\title{
TWISTED ACTION OF THE SYMMETRIC GROUP ON THE COHOMOLOGY OF A FLAG MANIFOLD
}

\author{
ALAIN LASCOUX \\ L.I.T.P., Université Paris 7 \\ 2, Place Jussieu, 75251 Paris Cedex 05, France \\ E-mail: al@litp.ibp.fr \\ BERNARD LECLERC \\ L.I.T.P., Université Paris 7 \\ 2, Place Jussieu, 75251 Paris Cedex 05, France \\ E-mail: bl@litp.ibp.fr \\ JEAN-YVES THIBON \\ Institut Gaspard Monge, Université de Marne-la-Vallée \\ 2, rue de la Butte-Verte, 93166 Noisy-le-Grand Cedex, France \\ E-mail: jyt@litp.ibp.fr
}

\begin{abstract}
Classes dual to Schubert cycles constitute a basis on the cohomology ring of the flag manifold $\mathcal{F}$, self-adjoint up to indexation with respect to the intersection form. Here, we study the bilinear form

$$
(X, Y):=\langle X \cdot Y, c(\mathcal{F})\rangle
$$

where $X, Y$ are cocycles, $c(\mathcal{F})$ is the total Chern class of $\mathcal{F}$ and $\langle$,$\rangle is the intersection form.$ This form is related to a twisted action of the symmetric group of the cohomology ring, and to the degenerate affine Hecke algebra. We give a distinguished basis for this form, which is a deformation of the usual basis of Schubert polynomials, and apply it to the computation of the Schubert cycle expansions of Chern classes of flag manifolds.
\end{abstract}

1. Introduction and preliminaries. Let $V$ be a complex vector space of dimension $n$, and $\mathcal{F}=\mathcal{F}(V)$ be the variety of complete flags in $V$. It is well known that the cohomology ring $H^{*}(\mathcal{F}, \mathbb{C})$ is the quotient of the polynomial ring $\mathbb{C}[X]=\mathbb{C}\left[x_{1}, x_{2}, \ldots, x_{n}\right]$ by the ideal $\mathcal{I}^{+}$of symmetric polynomials without constant term.

Let $\sigma_{i}, i=1, \ldots, n-1$ be the simple transposition exchanging $x_{i}$ and $x_{i+1}$. Denote

1991 Mathematics Subject Classification: 14M15, 05E15, 20G20.

Supported by PRC Math-Info and EEC grant $n^{0}$ ERBCHRXCT930400.

The paper is in final form and no version of it will be published elsewhere. 
by $\partial_{i}$ the linear operator on $\mathbb{C}\left[x_{1}, \ldots, x_{n}\right]$ defined by

$$
\partial_{i} f:=\frac{f-\sigma_{i} f}{x_{i}-x_{i+1}}
$$

(Newton's divided difference). The operators $\partial_{1}, \ldots, \partial_{n-1}$ induce operators on $H^{*}(\mathcal{F})$.

According to [1] and [4], the basis of Schubert cycles can be obtained from the class of a point $P=\frac{1}{n !} \prod_{i<j}\left(x_{i}-x_{j}\right)$ by successive applications of divided difference operators. Taking as representative of $P$ the polynomial $X:=x_{1}^{n-1} x_{2}^{n-2} \cdots x_{1}^{0}$, one obtains polynomials $X_{\mu}, \mu \in \mathfrak{S}_{n}$, called Schubert polynomials, which represent the Schubert subvarieties in the cohomology ring [11]. A detailed account of the algebraic theory of Schubert polynomials can be found in Macdonald's treatise [14].

Divided differences satisfy the braid relations

$$
\left\{\begin{aligned}
\partial_{i} \partial_{i+1} \partial_{i} & =\partial_{i+1} \partial_{i} \partial_{i+1} \\
\partial_{i} \partial_{j} & =\partial_{j} \partial_{i} \quad \text { for } \quad|i-j|>1,
\end{aligned}\right.
$$

but the squares $\partial_{i}^{2}$ are null. These relations allow to define operators $\partial_{\mu}$ for any permutation $\mu \in \mathfrak{S}_{n}$ : if $\mu=\sigma_{i_{1}} \sigma_{i_{2}} \cdots \sigma_{i_{m}}$ is a reduced decomposition of $\mu$, one sets $\partial_{\mu}=\partial_{i_{1}} \partial_{i_{2}} \cdots \partial_{i_{m}}$. The result does not depend on the choice of a particular reduced decomposition of $\mu$.

To recover an action of the symmetric group, one can take any $q \in \mathbb{C}$ and define

$$
D_{i}:=\sigma_{i}+q \partial_{i}, \quad 1 \leq i \leq n-1 .
$$

These operators still satisfy the braid relations

$$
\left\{\begin{aligned}
D_{i} D_{i+1} D_{i} & =D_{i+1} D_{i} D_{i+1} \\
D_{i} D_{j} & =D_{j} D_{i} \quad(|i-j|>1)
\end{aligned}\right.
$$

together with

$$
D_{i}^{2}=1
$$

so that they generate a representation of the symmetric group $\mathfrak{S}_{n}$ on the polynomial ring $\mathbb{C}\left[x_{1}, \ldots, x_{n}\right]$, as well as on the cohomology ring $H^{*}(\mathcal{F}, \mathbb{C})$. These operators have been considered by Cherednik and Bernstein (cf. [2], [3]). Similar operators, acting on the equivariant $K$-theory of flag manifolds, have been used by Lusztig [13]. More general operators satisfying braid relations have been given in [12].

As $\partial_{i}$ decreases degrees by 1 , all $q \neq 0$ will give equivalent representations of $\mathfrak{S}_{n}$, and by homogeneity, the general case can be recovered from the case $q=1$. For simplicity, we set $q=1$, and write

$$
s_{i}:=\sigma_{i}+\partial_{i} .
$$

We denote as above by $s_{\mu}$ the product of operators $s_{i}$ corresponding to a permutation $\mu$. Remark that the operator algebra generated by the $s_{i}$ and the variables $x_{j}$ (interpreted as operators $\left.f \mapsto x_{j} f\right)$ is isomorphic to the degenerate affine Hecke algebra considered in [2].

Schubert calculus for other classical groups can be found in the work of Fulton [7] and of Pragacz and Ratajski [15].

This paper is organized as follows. We first define certain elements (Yang-Baxter operators) of the degenerate affine Hecke algebra. Then we use them to define a bilinear form on the cohomology of a flag manifold. We exhibit a distinguished basis, called affine 
Schubert polynomials, and compute its adjoint basis. We then apply this formalism to the computation of the Schubert expansions of Chern classes.

Acknowledgements. The preparation of this paper has been facilitated by the use of the program system SYMMETRICA [9] and of the Maple package SP [16].

2. Yang-Baxter operators. We shall define inductively operators $\square_{\mu}$ and $\nabla_{\mu}$ associated with any permutation $\mu$ in $\mathfrak{S}_{n}$. Set $\square_{12 \ldots n}=1, \nabla_{12 \ldots n}=1$, and, if $\mu=\sigma_{i} \alpha$ with $\ell(\mu)=\ell(\alpha)+1$, and $\beta=\alpha^{-1}$,

$$
\left\{\begin{array}{l}
\square_{\mu}=\left(s_{i}+\frac{1}{\beta_{i+1}-\beta_{i}}\right) \square_{\alpha} \\
\nabla_{\mu}=\left(s_{i}-\frac{1}{\beta_{i+1}-\beta_{i}}\right) \nabla_{\alpha}
\end{array}\right.
$$

Using the braid relations (4), one can check that this definition is consistent, i.e. does not depend on the chosen factorization (see $[2,3]$ and [6]). This follows in fact from a classical solution of the Yang-Baxter equation. In [17], C. N. Yang observed that the operators defined by $Y_{i}(u)=u^{-1}+\sigma_{i}$, where $u$ is a scalar parameter and $\sigma_{i}$ the transposition $(i, i+1)$ satisfy the "Quantum Yang-Baxter Equation with spectral parameter":

$$
Y_{i}(u-v) Y_{i+1}(u-w) Y_{i}(v-w)=Y_{i+1}(v-w) Y_{i}(u-w) Y_{i+1}(u-v)
$$

It follows that given a $n$-tuple of parameters $\mathbf{u}=\left(u_{1}, \ldots, u_{n}\right)$, one can define for any permutation $\mu \in \mathfrak{S}_{n}$ an operator $R_{\mu}(\mathbf{u})$ by the following prescription: $Y_{\mu}(\mathbf{u})=Y_{i}\left(u_{\beta(i+1)}-\right.$ $\left.u_{\beta(i)}\right) R_{\alpha}(\mathbf{u})$, where, as above, $R_{12 \ldots n}=1, \mu=\sigma_{i} \alpha, \ell(\mu)=\ell(\alpha)+1$ and $\beta=\alpha^{-1}$. Then, our operators $(7)$ are respectively $R_{\mu}(\mathbf{u})$ and $R_{\mu}(-\mathbf{u})$, where $\mathbf{u}=(1,2, \ldots, n)$ and $\sigma_{i}$ is interpreted as $s_{i}$.

For the maximal element $\omega=(n, n-1, \ldots, 1)$ of $\mathfrak{S}_{n}$, one has the following factorization property (given in [6] for the case of the Hecke algebra):

Proposition 2.1. Define $\theta=\prod_{1 \leq i<j \leq n}\left(1+x_{i}-x_{j}\right)$ and $\theta^{*}=\prod_{1 \leq i<j \leq n}\left(1-x_{i}+x_{j}\right)$.

Then, for any polynomial $f$,

(i) $\nabla_{\omega} f=\theta^{*} \partial_{\omega} f$

(ii) $\square_{\omega} f=\partial_{\omega}(\theta f)$.

Pr o of. Recall that the classes of the Schubert polynomials $X_{\mu}, \mu \in \mathfrak{S}_{n}$, form a basis of $H^{*}(\mathcal{F})=\mathbb{C}[X] / \mathcal{I}^{+}$. Given $\mu$ and $i$ such that $\ell\left(\mu \sigma_{i}\right)>\ell(\mu)$, the polynomial $X_{\mu}$ is symmetrical in $x_{i}$ and $x_{i+1}$. As such, it is sent to 0 by the operator $\nabla_{\sigma_{i}}=\sigma_{i}+\partial_{i}-1$.

Now, for any permutation $\mu \neq \omega$, there exists an $i$ such that $\ell\left(\mu \sigma_{i}\right)>\ell(\mu)$. If we choose a reduced decomposition of $\omega$ ending by $\sigma_{i}, \omega=\nu \sigma_{i}$, say, we see that $X_{\mu}$ is sent to 0 by $\partial_{\omega}=\partial_{\nu} \partial_{\sigma_{i}}$ and by $\nabla_{\omega}=\nabla_{\mu} \nabla_{\sigma_{i}}$.

Thus, $\nabla_{\omega}$ as well as $\partial_{\omega}$ annihilate all Schubert polynomials $X_{\mu}$ for $\mu \neq \omega$. Finally, $X_{\omega}=x_{1}^{n-1} \ldots x_{n}^{0}$ is sent to 1 by $\partial_{\omega}$. To conclude, it remains to prove that

$$
\nabla_{\omega}\left(X_{\omega}\right)=\prod_{1 \leq i<j \leq n}\left(1-x_{i}+x_{j}\right)
$$

This formula can be proved by induction on $n$ using the factorization

$$
\omega_{n}=\sigma_{1} \sigma_{2} \cdots \sigma_{n-1} \omega_{n-1},
$$


which gives

$$
\nabla_{\omega_{n}}=\left(s_{1}-1\right) \cdots\left(s_{n-1}-\frac{1}{n-1}\right) \nabla_{\omega_{n-1}}
$$

3. Quadratic form. Recall that the intersection form of the cohomology ring $H^{*}(\mathcal{F}, \mathbb{C})$ is induced by the form on $\mathbb{C}[X]$

$$
\langle f, g\rangle=\left.\partial_{\omega}(f g)\right|_{0}=\partial_{\omega}\left(\left.f g\right|_{\ell(\omega)}\right)
$$

where $\left.f\right|_{k}$ denotes the homogeneous component of degree $k$ of $f$ (cf. [1], [5]). With respect to this form, the Schubert polynomials satisfy

$$
\left\langle X_{\mu}, X_{\nu}\right\rangle= \begin{cases}1 & \text { if } \nu=\omega \mu \\ 0 & \text { otherwise }\end{cases}
$$

The tangent bundle $T \mathcal{F}$ of the flag manifold has a composition sequence $\left\{L_{i} L_{j}^{-1}\right\}_{i<j}$ where $L_{1}, L_{2}, \ldots, L_{n}$ are the tautological line bundles on $\mathcal{F}$. The total Chern class of $L_{i}$ being $c\left(L_{i}\right)=1+x_{i}$, the total Chern class of the tangent bundle of $\mathcal{F}$ is

$$
c(\mathcal{F})=\prod_{i<j}\left(1+x_{i}-x_{j}\right)
$$

(see e.g. [8], our convention is $L_{i}=\xi_{i}^{*}$ in the notation of [8]). Consider now the following quadratic form on $\mathbb{C}[X]$ :

DEFINITION 3.1.

$$
(f, g):=\left.\square_{\omega}(f g)\right|_{0} .
$$

Thus, in the cohomology ring, we see from Proposition 2.1 that

$$
(f, g)=\langle f, g c(\mathcal{F})\rangle=\langle f c(\mathcal{F}), g\rangle .
$$

Lemma 3.2. The operators $\square_{i}$ are self-adjoint with respect to the quadratic form (, ).

Proof. For any $i, \square_{\omega} \square_{i}=2 \square_{\omega}$, since $\square_{i}^{2}=2 \square_{i}$ and since one can find a reduced decomposition of $\omega$ ending with $\sigma_{i}$. Now,

$$
\left(\square_{i} f, g\right)=\left.\square_{\omega}\left(\left(\square_{i} f\right) g\right)\right|_{0}=\left.\frac{1}{2} \square_{\omega} \square_{i}\left(\left(\square_{i} f\right) g\right)\right|_{0}=\left.\frac{1}{2} \square_{\omega}\left(\left(\square_{i} f\right)\left(\square_{i} g\right)\right)\right|_{0}
$$

since $\square_{i} f$ is a scalar for $\square_{i}$, being symmetrical in $x_{i}, x_{i+1}$. The last expression being symmetrical in $f, g$, this proves that $\left(\square_{i} f, g\right)=\left(f, \square_{i} g\right)$.

4. Affine Schubert polynomials. Let $\mathcal{H}_{n}$ be the linear subspace of $\mathbb{C}\left[x_{1}, \ldots, x_{n}\right]$ generated by the monomials $x^{I}=x_{1}^{i_{1}} x_{2}^{i_{2}} \cdots x_{n}^{i_{n}}$ such that $i_{k} \leq n-k$. Let $\Pi$ be the projector from $\mathbb{C}[X]$ onto $\mathcal{H}_{n}$ associating to a polynomial $P$ the unique representative in $\mathcal{H}_{n}$ of its class $\bar{P} \in \mathbb{C}[X] / \mathcal{I}^{+}$.

Definition 4.1. Let $\mu \in \mathfrak{S}_{n}$. The affine Schubert polynomial of index $\mu$ is defined by

$$
Z_{\mu}=\Pi\left(\square_{\mu^{-1} \omega} Z_{\omega}\right)
$$

where $Z_{\omega}:=X_{\omega}=x_{1}^{n-1} x_{2}^{n-2} \cdots x_{n}^{0}$. 
EXAMPLE 4.2. For $n=3$,

$$
\begin{aligned}
Z_{321} & =x_{1}^{2} x_{2} \\
Z_{312} & =x_{1}^{2} \\
Z_{231} & =x_{1} x_{2} \\
Z_{213} & =x_{1}-1 / 2 x_{1} x_{2}-x_{1}^{2} \\
Z_{132} & =x_{1}+x_{2}-x_{1} x_{2}-1 / 2 x_{1}^{2} \\
Z_{123} & =1
\end{aligned}
$$

In general, one has $Z_{\omega}=X_{\omega}, Z_{\mu}=X_{\mu}+$ (terms of degree $\left.>\ell(\mu)\right)$, and $Z_{i d}=1$, the last identity being due to the fact that $\square_{\omega}\left(Z_{\omega}\right)$ is symmetrical with term of lowest degree $X_{i d}=1$.

EXAMPLE 4.3. For $n=3$,

$$
\begin{aligned}
& Z_{321}=X_{321} \\
& Z_{312}=X_{312} \\
& Z_{231}=X_{231} \\
& Z_{213}=X_{213}-1 / 2 X_{231}-X_{312} \\
& Z_{132}=X_{132}-X_{231}-1 / 2 X_{312} \\
& Z_{123}=X_{123}
\end{aligned}
$$

THEOREM 4.4. The polynomials $Z_{\mu}, \mu \in \mathfrak{S}_{n}$, form a basis of $\mathcal{H}_{n}$. The quadratic form $\left(\right.$, ) is positive definite, and the adjoint basis of $\left\{Z_{\mu}\right\}$ is $\left\{Z_{\mu}^{\vee}\right\}$ where $Z_{\mu}^{\vee}=\Pi\left(\nabla_{\mu^{-1} \omega} X_{\omega}\right)$.

Proof. $Z_{\mu}$ is a non-homogeneous polynomial with the Schubert polynomial $X_{\mu}$ as its term of smallest degree. Since the classes of the Schubert polynomials form a basis of $H^{*}(\mathcal{F})$, the same is true for the $Z_{\mu}$.

The polynomials $Z_{\omega}^{\vee} Z_{\mu} \theta$ (for $\mu \neq i d$ ) have no component of degree $\ell(\omega)$. Therefore, their images under $\partial_{\omega}$ are symmetric polynomials without constant term, which proves that for all $\mu \neq i d,\left(Z_{\omega}^{\vee}, Z_{\mu}\right)=0$. On the other hand,

$$
\left(Z_{\omega}^{\vee}, Z_{12 \ldots n}\right)=\left(Z_{\omega}^{\vee}, 1\right)=\left.\partial_{\omega}\left(X_{\omega} \theta\right)\right|_{0}=\partial_{\omega}\left(\left.\left(X_{\omega} \theta\right)\right|_{\ell(\omega)}\right)=\partial_{\omega} X_{\omega}=X_{12 \ldots n}=1 .
$$

For the general case of a $Z_{\nu}^{\vee}$, one uses induction on the length of $\nu$. Let $\nu$ and $i$ be such that $\ell\left(\nu \sigma_{i}\right)<\ell(\nu)$. Then, for any $\mu$ and an appropriate constant $k$

$$
\left(Z_{\mu}, Z_{\nu}^{\vee}\right)=\left(Z_{\mu},\left(s_{i}-k\right) Z_{\nu}^{\vee}\right)=\left(\left(s_{i}-k\right) Z_{\mu}, Z_{\nu}^{\vee}\right)=k^{\prime}\left(Z_{\mu}, Z_{\nu}^{\vee}\right)+k^{\prime \prime}\left(Z_{\mu \sigma_{i}}, Z_{\nu}^{\vee}\right)
$$

(for some other scalars $\left.k^{\prime}, k^{\prime \prime}\right)$. By induction, one can suppose $\left(Z_{\mu}, Z_{\nu}^{\vee}\right)=0$ for $\mu \nu^{-1} \neq \omega$.

One is thus reduced to study the case

$$
\mu \sigma_{i} \nu^{-1}=\omega, \quad \ell\left(\mu \sigma_{i}\right)>\ell(\mu)
$$

In that case,

$$
Z_{\mu \sigma_{i}}=\left(s_{i}+\frac{1}{r}\right) Z_{\mu} \text { and } Z_{\mu \sigma_{i}}^{\vee}=\left(s_{i}-\frac{1}{r}\right) Z_{\mu}^{\vee}
$$

for a certain integer $r$. Then, we check

$$
\left(Z_{\mu \sigma_{i}}, Z_{\nu \sigma_{i}}^{\vee}\right)=\left(\left(s_{i}+\frac{1}{r}\right) Z_{\mu},\left(s_{i}-\frac{1}{r}\right) Z_{\mu}^{\vee}\right)=\left(\left(s_{i}^{2}-\frac{1}{r^{2}}\right) Z_{\mu}, Z_{\nu}^{\vee}\right)=0,
$$


and

$$
\left(Z_{\mu}, Z_{\nu \sigma_{i}}^{\vee}\right)=\left(\left(s_{i}+\frac{1}{r}-\frac{2}{r}\right) Z_{\mu}, Z_{\nu}^{\vee}\right)=\left(Z_{\mu \sigma_{i}}, Z_{\nu}^{\vee}\right)-\frac{2}{r}\left(Z_{\mu}, Z_{\nu}^{\vee}\right)=1-0 .
$$

EXAMPLE 4.5. Again for $n=3$,

$$
\begin{aligned}
& Z_{321}^{\vee}=x_{1}^{2} x_{2} \\
& Z_{312}^{\vee}=x_{1}^{2}-2 x_{1}^{2} x_{2} \\
& Z_{231}^{\vee}=x_{1} x_{2}-2 x_{1}^{2} x_{2} \\
& Z_{213}^{\vee}=x_{1}-3 / 2 x_{1} x_{2}-3 x_{1}^{2}+3 x_{1}^{2} x_{2} \\
& Z_{132}^{\vee}=x_{1}+x_{2}-3 x_{1} x_{2}-3 / 2 x_{1}^{2}+3 x_{1}^{2} x_{2} \\
& Z_{123}^{\vee}=1-4 x_{1}-2 x_{2}+6 x_{1} x_{2}+6 x_{1}^{2}-6 x_{1}^{2} x_{2}
\end{aligned}
$$

5. Change of basis. The operators $\partial_{i}$ are self-adjoint with respect to $\langle$,$\rangle , but \sigma_{i}$ is adjoint to $-\sigma_{i}$. This implies that $-s_{i}$ is adjoint to $\bar{s}_{i}:=\sigma_{i}-\partial_{i}$.

Let us define $\bar{\square}_{\mu}, \bar{\nabla}_{\mu}$ to be the images of $\square_{\mu}$ and $\nabla_{\mu}$ under the replacement $s_{i} \mapsto \bar{s}_{i}$. We also define

$$
\bar{Z}_{\mu}:=(-1)^{\ell(\omega \mu)} \Pi\left(\bar{\square}_{\mu^{-1} \omega} Z_{\omega}\right), \quad \bar{Z}_{\mu}^{\vee}:=(-1)^{\ell(\omega \mu)} \Pi\left(\bar{\nabla}_{\mu^{-1} \omega} Z_{\omega}\right) .
$$

Then, $(-1)^{\ell(\mu)} \bar{Z}_{\mu}$ is obtained from $Z_{\mu}$ under the transformation $x_{i} \mapsto-x_{i}$, since signs in the expansion of $Z_{\mu}$ correspond to the degree.

LEMMA 5.1. $\left\{\bar{Z}_{\omega \mu}\right\}$ is the adjoint basis of $\left\{Z_{\mu}\right\}$ with respect to $\langle$, $\rangle$, i.e. one has $\left\langle\bar{Z}_{\omega \mu}, Z_{\mu}\right\rangle=1$ and $\left\langle\bar{Z}_{\omega \mu}, Z_{\nu}\right\rangle=0$ for $\nu \neq \mu$.

Similarly, $\left\{\bar{Z}_{\omega \mu}^{\vee}\right\}$ is the adjoint basis of $\left\{Z_{\mu}^{\vee}\right\}$ for $\langle$,$\rangle .$

Proof. As in Section 4, the lemma is proved by induction on the length of $\mu$, starting from the case

$$
\left\langle Z_{\omega \mu}, \bar{Z}_{\omega}\right\rangle=0 \text { if } \mu \neq \omega
$$

Take $i$ such that $\ell\left(\mu \sigma_{i}\right)>\ell(\mu)$. Then,

$$
\left\langle Z_{\omega \mu \sigma_{i}}, \bar{Z}_{\nu}\right\rangle=\left\langle\left(s_{i}+\frac{1}{r}\right) Z_{\omega \mu}, \bar{Z}_{\nu}\right\rangle=\left\langle Z_{\omega \mu},\left(-\bar{s}_{i}+\frac{1}{r}\right) \bar{Z}_{\nu}\right\rangle .
$$

Since $\left(-\bar{s}_{i}+\frac{1}{r}\right) \bar{Z}_{\nu}$ is a linear combination of $\bar{Z}_{\nu}$ and $\bar{Z}_{\nu \sigma_{i}}$, the nullity of the scalar products $\left\langle Z_{\omega \mu \sigma_{i}}, \bar{Z}_{\nu}\right\rangle$ follows from those of $\left\langle Z_{\omega \mu}, Z_{\nu}\right\rangle$ for $\nu \neq \mu$ and $\nu \neq \mu \sigma_{i}$. In the special case $\nu=\mu \sigma_{i}$, one has

$$
\left\langle Z_{\omega \mu \sigma_{i}}, \bar{Z}_{\mu}\right\rangle=\left\langle Z_{\omega \mu},\left(-\bar{s}_{i}+\frac{1}{r}\right)\left(-\bar{s}_{i}-\frac{1}{r}\right) Z_{\mu \sigma_{i}}\right\rangle=\left\langle Z_{\omega \mu},\left(1-\frac{1}{r^{2}}\right) Z_{\mu \sigma_{i}}\right\rangle
$$

which is null.

Example 5.2.

$$
\begin{aligned}
\left\langle Z_{23514}, \bar{Z}_{41352}\right\rangle & =\left\langle\left(s_{2}+\frac{1}{2}\right) Z_{25314},\left(-\bar{s}_{2}-\frac{1}{2}\right) \bar{Z}_{43152}\right\rangle \\
& =\left\langle\left(s_{2}-\frac{1}{2}\right)\left(s_{2}+\frac{1}{2}\right) Z_{25314}, \bar{Z}_{43152}\right\rangle \\
& =\left\langle\left(1-\frac{1}{4}\right) Z_{25314}, \bar{Z}_{43152}\right\rangle=0 .
\end{aligned}
$$


Let $\left\{A_{\mu}\right\}$ and $\left\{B_{\nu}\right\}$ be two bases of $\mathcal{H}_{n}$. We denote by $M(A, B)$ the transition matrix from the basis $\left\{A_{\mu}\right\}$ to the basis $\left\{B_{\nu}\right\}$, with the convention

$$
A_{\mu}=\sum_{\nu} M(A, B)_{\mu \nu} B_{\nu} .
$$

For example, $M(Z, X)_{\mu \nu}=\left\langle Z_{\mu}, X_{\omega \nu}\right\rangle$ and $M(X, Z)_{\mu \nu}=\left(X_{\mu}, Z_{\omega \nu}^{\vee}\right)$. These matrices have a symmetry property, thanks to the following property of the scalar product:

$$
\langle\omega P, \omega Q\rangle=(-1)^{\ell(\omega)}\langle P, Q\rangle .
$$

Indeed, taking into account the two identities

$$
\omega\left(X_{\mu}\right)=(-1)^{\ell(\mu)} X_{\omega \mu \omega}, \quad \omega\left(Z_{\mu}\right)=(-1)^{\ell(\mu)} \bar{Z}_{\omega \mu \omega}
$$

we see that the four matrices

$$
M(Z, X), M(X, Z), M\left(Z^{\vee}, X\right) \text { and } M\left(X, Z^{\vee}\right)
$$

possess the symmetry

$$
M_{\mu \nu}=M_{\omega \mu \omega, \omega \nu \omega} .
$$

Furthermore, we have the following relation between these matrices and their inverses:

THEOREM 5.3. The inverse of $M(Z, X)$ is a matrix with nonnegative entries, given by

Similarly,

$$
M(X, Z)_{\mu \nu}=\left|M(Z, X)_{\nu \omega, \mu \omega}\right| .
$$

where $|\cdot|$ denotes the absolute value.

$$
M\left(X, Z^{\vee}\right)_{\mu \nu}=\left|M\left(Z^{\vee}, X\right)_{\nu \omega, \mu \omega}\right|,
$$

Proof. The first matrix corresponds to the expansions

$$
Z_{\mu}=\sum_{\nu}\left\langle Z_{\mu}, X_{\omega \nu}\right\rangle X_{\nu}
$$

The inverse formulas are, according to Lemma 5.1,

$$
X_{\nu}=\sum_{\mu}\left\langle\bar{Z}_{\omega \mu}, X_{\nu}\right\rangle Z_{\mu} .
$$

But now, $\left\langle\bar{Z}_{\omega \mu}, X_{\nu}\right\rangle=\left|\left\langle Z_{\omega \mu}, X_{\nu}\right\rangle\right|$, whence the first part of the theorem follows. The proof of the second part is similar.

Thus, the inverse of the matrix $M(Z, X)$ is obtained from $M(Z, X)$ by reflection through the antidiagonal $(\mu, \nu) \longrightarrow(\omega \nu, \omega \mu)$ and suppression of the signs.

Corollary 5.4. For any pair of permutations,

$$
\left(X_{\mu}, Z_{\eta}^{\vee}\right)=\left|\left\langle X_{\omega \mu \omega}, Z_{\omega \eta \omega}\right\rangle\right|
$$

and

$$
\left(X_{\mu}, Z_{\eta}\right)=\left|\left\langle X_{\omega \mu \omega}, Z_{\omega \eta \omega}^{\vee}\right\rangle\right| .
$$

Indeed,

$$
X_{\mu}=\sum_{\eta}\left(X_{\mu}, Z_{\eta}^{\vee}\right) Z_{\omega \eta}
$$

but we have just seen that the coefficients of the expansion of $X_{\mu}$ in the basis $Z_{\omega \eta}$ are the $\left\langle\bar{Z}_{\eta}, X_{\mu}\right\rangle$. 
6. Schubert expansions of Chern classes. Let $|I|$ be a composition of $n$, i.e. $I \in \mathbb{N}^{r}$ with $|I|:=i_{1}+\ldots+i_{r}=n$, and let $J_{1}, \ldots, J_{r}$ be the associated decomposition of the interval $[1, n]$, that is

$$
J_{1}=\left[1, i_{1}\right], \quad J_{2}=\left[i_{1}+1, i_{1}+i_{2}\right], \quad \ldots, \quad J_{r}=\left[i_{1}+\cdots+i_{r-1}+1, n\right] .
$$

Let $\mathcal{F}_{I}$ be the variety of flags

$$
V_{0}=\{0\} \subset V_{1} \subset V_{2} \subset \ldots \subset V_{r}=V
$$

such that $\operatorname{dim} V_{k}=i_{1}+\cdots+i_{k}$. Let also $\mathfrak{S}_{I}$ denote the Young subgroup

$$
\mathfrak{S}\left(J_{1}\right) \times \mathfrak{S}\left(J_{2}\right) \times \cdots \times \mathfrak{S}\left(J_{r}\right) \subset \mathfrak{S}_{n}
$$

associated to the composition $I$. The Chern class $\theta^{I}$ of the tangent bundle of $\mathcal{F}_{I}$ is the maximal $\mathfrak{S}_{I}$-invariant factor of $\theta$ :

$$
\theta^{I}=\theta /\left(\theta_{J_{1}} \theta_{J_{2}} \cdots \theta_{J_{r}}\right)
$$

where

$$
\theta_{J_{k}}=\prod_{\substack{i<j \\ i, j \in J_{k}}}\left(1+x_{i}-x_{j}\right) .
$$

A basis of the cohomology ring $H^{*}\left(\mathcal{F}_{I}\right)$ is the set of Schubert polynomials $X_{\mu}$ with $\mu$ minimal in its right coset $\mu \mathfrak{S}_{I}$. In other words, one restricts the Schubert basis $\left(X_{\mu}\right)$ to those $\mu$ such that $\mu_{1}<\ldots<\mu_{i_{1}}, \mu_{i_{1}+1}<\ldots<\mu_{i_{1}+i_{2}}, \ldots, \mu_{i_{1}+\ldots+i_{r-1}+1}<\ldots<\mu_{n}$. Since $\mu_{i}<\mu_{i+1}$ iff $X_{\mu}$ is symmetrical in $x_{i}$ and $x_{i+1}$, the Schubert basis of $H^{*}\left(\mathcal{F}_{I}\right)$ consists of those Schubert polynomials which are invariant under $\mathfrak{S}_{I}$.

Define the Chern coefficient $c_{\mu}\left[\mathcal{F}_{I}\right]$ of the variety $\mathcal{F}_{I}$ as the coefficient of (the class) of $X_{\mu}$ in the expansion of $\theta^{I}$ on the Schubert basis of $H^{*}\left(\mathcal{F}_{I}\right)$. In other words,

$$
c_{\mu}\left[\mathcal{F}_{I}\right]=\left\langle\theta^{I}, X_{\omega \mu}\right\rangle .
$$

As in the case of the full flag variety $\mathcal{F}$, these scalar products can be computed with the help of the scalar product $($,$) .$

Let $\omega_{I}$ be the maximal element of $\mathfrak{S}_{I}$, and $\zeta_{I}:=\omega_{I} \omega$. We have seen that

$$
\square_{\omega}=\partial_{\omega} \theta=\partial_{\zeta_{I}} \partial_{\omega_{I}} \theta^{I} \theta_{J_{1}} \theta_{J_{2}} \cdots \theta_{J_{r}} .
$$

The operator $\partial_{\omega}$ factorizes

$$
\partial_{\omega}=\partial_{\zeta_{i}} \partial_{\omega_{I}}
$$

so that

$$
\begin{gathered}
c_{\mu}\left[\mathcal{F}_{I}\right]=\partial_{\omega}\left(\theta^{I} X_{\omega \mu}\right) \\
=\partial_{\zeta_{i}} \partial_{\omega_{i}}\left(\theta^{I} X_{\omega \mu}\right)=\partial_{\zeta_{i}}\left(\theta^{I} \partial_{\omega_{i}} X_{\omega \mu}\right) \\
=\partial_{\zeta_{I}}\left(\theta^{I} X_{\omega \mu \omega_{I}}\right)=\partial_{\zeta_{i}}\left(\theta^{I} X_{\omega \mu \omega_{I}}\right)\left(\partial_{\omega_{I}} \theta_{J_{1}} \theta_{J_{2}} \cdots \theta_{J_{r}} / I !\right) \\
=\frac{1}{I !} \partial_{\omega}\left(\theta X_{\omega \mu \omega_{I}}\right) \\
=\frac{1}{I !}\left(1, X_{\omega \mu \omega_{I}}\right)
\end{gathered}
$$

Equality (18) follows from the fact that $\theta^{I}$ is invariant under $\mathfrak{S}_{I}$ and thus commutes with $\partial_{\omega_{I}}$. Now, $\theta$ is of degree $\left(\begin{array}{l}n \\ 2\end{array}\right)$, and $\partial_{\omega}$ decreases degrees by $\ell(\omega)=\left(\begin{array}{l}n \\ 2\end{array}\right)$. Thus $\partial_{\omega}(\theta)$ 
is a scalar which is checked to be $n$ !. More generally, by direct product, one has for the maximal element of the Young subgroup $\mathfrak{S}_{I}$

$$
\partial_{\omega_{I}} \theta_{J_{1}} \cdots \theta_{J_{r}}=I !:=i_{1} ! \cdots i_{r} !
$$

and equality (19) follows from this identity.

Since $\theta^{I}$ as well as $X_{\omega \mu \omega_{I}}$ are invariant under $\mathfrak{S}_{I}$, they commute with $\partial_{\omega_{I}}$, which is step (20).

Summarizing, we have the following expression for the components of the Chern class of $\mathcal{F}_{I}$ on the Schubert basis.

TheOREM 6.1. Let $I=\left(i_{1}, \ldots, i_{r}\right)$ be a composition of $n, \mathfrak{S}_{I}$ and $\mathcal{F}_{I}$ the corresponding Young subgroup and flag variety. Let $\mu$ be a permutation which is minimum in its coset $\mu \mathfrak{S}_{I}$. Then, the Chern coefficient $c_{\mu}\left[\mathcal{F}_{I}\right]$ is given by

$$
c_{\mu}\left[\mathcal{F}_{I}\right]=\left(1, X_{\omega \mu \omega_{I}}\right) / I !
$$

In particular, for the full flag variety (case $I=(1,1, \ldots, 1)$ ), one has

$$
c_{\mu}[\mathcal{F}]=\left(1, X_{\omega \mu}\right)=\square_{\omega}\left(X_{\omega \mu}\right)
$$

and these numbers constitute the first column of the matrix $M\left(X, Z^{\vee}\right)$. Equivalently, they are equal to the absolute values of the entries of the last row of $M\left(Z^{\vee}, X\right)$.

In the case of a Grassmann manifold $G(p, p+q)=\mathcal{F}_{(p, q)}$, the basis of $H^{*}\left(\mathcal{F}_{(p, q)}\right)$ consists of those $X_{\mu}$ for which $\mu_{1}<\ldots<\mu_{p}$ and $\mu_{p+1}<\ldots<\mu_{p+q}$ (Grassmannian permutations). In fact, for such a permutation, $X_{\mu}$ is equal to the Schur function indexed by the partition $\left(\mu_{1}-1, \mu_{2}-2, \ldots, \mu_{p}-p\right)$ on the set of variables $\left\{x_{1}, \ldots, x_{p}\right\}$. Thus, the Chern coefficient $c_{\mu}\left[\mathcal{F}_{(p, q)}\right]$ is

$$
c_{\mu}\left[\mathcal{F}_{(p, q)}\right]=\square_{\omega}\left(X_{\left(n+1-\mu_{p}, \ldots, n+1-\mu_{1}, n+1-\mu_{n}, \ldots, n+1-\mu_{p+1}\right)}\right) .
$$

For example, up to a factor $(2 !)^{2}$, the Chern coefficients of $\mathcal{F}_{(2,2)}$ are $4,16,28,28,48,24$. They are given by the absolute values of the six entries of the bottom row of the matrix $M\left(Z^{\vee}, X\right)$ corresponding to columns indexed by permutations $\omega \mu$ where $\mu$ is Grassmannian.

7. Tables for $n=4$.

7.1. Affine Schubert polynomials.

$Z_{4321}=x_{1}^{3} x_{2}^{2} x_{3}$

$Z_{4312}=x_{1}^{3} x_{2}^{2}$

$Z_{4231}=x_{1}^{3} x_{2} x_{3}$

$Z_{4213}=x_{1}^{3} x_{2}-1 / 2 x_{1}^{3} x_{2} x_{3}-x_{1}^{3} x_{2}^{2}$

$Z_{4132}=x_{1}^{3} x_{3}+x_{1}^{3} x_{2}-x_{1}^{3} x_{2} x_{3}-1 / 2 x_{1}^{3} x_{2}^{2}$

$Z_{4123}=x_{1}^{3}$

$Z_{3421}=x_{1}^{2} x_{2}^{2} x_{3}$

$Z_{3412}=x_{1}^{2} x_{2}^{2}$

$Z_{3241}=x_{1}^{2} x_{2} x_{3}-1 / 2 x_{1}^{2} x_{2}^{2} x_{3}-x_{1}^{3} x_{2} x_{3}$

$Z_{3214}=x_{1}^{2} x_{2}-2 / 3 x_{1}^{2} x_{2} x_{3}-3 / 2 x_{1}^{2} x_{2}^{2}+1 / 3 x_{1}^{2} x_{2}^{2} x_{3}-2 x_{1}^{3} x_{2}+2 / 3 x_{1}^{3} x_{2} x_{3}+x_{1}^{3} x_{2}^{2}$ 


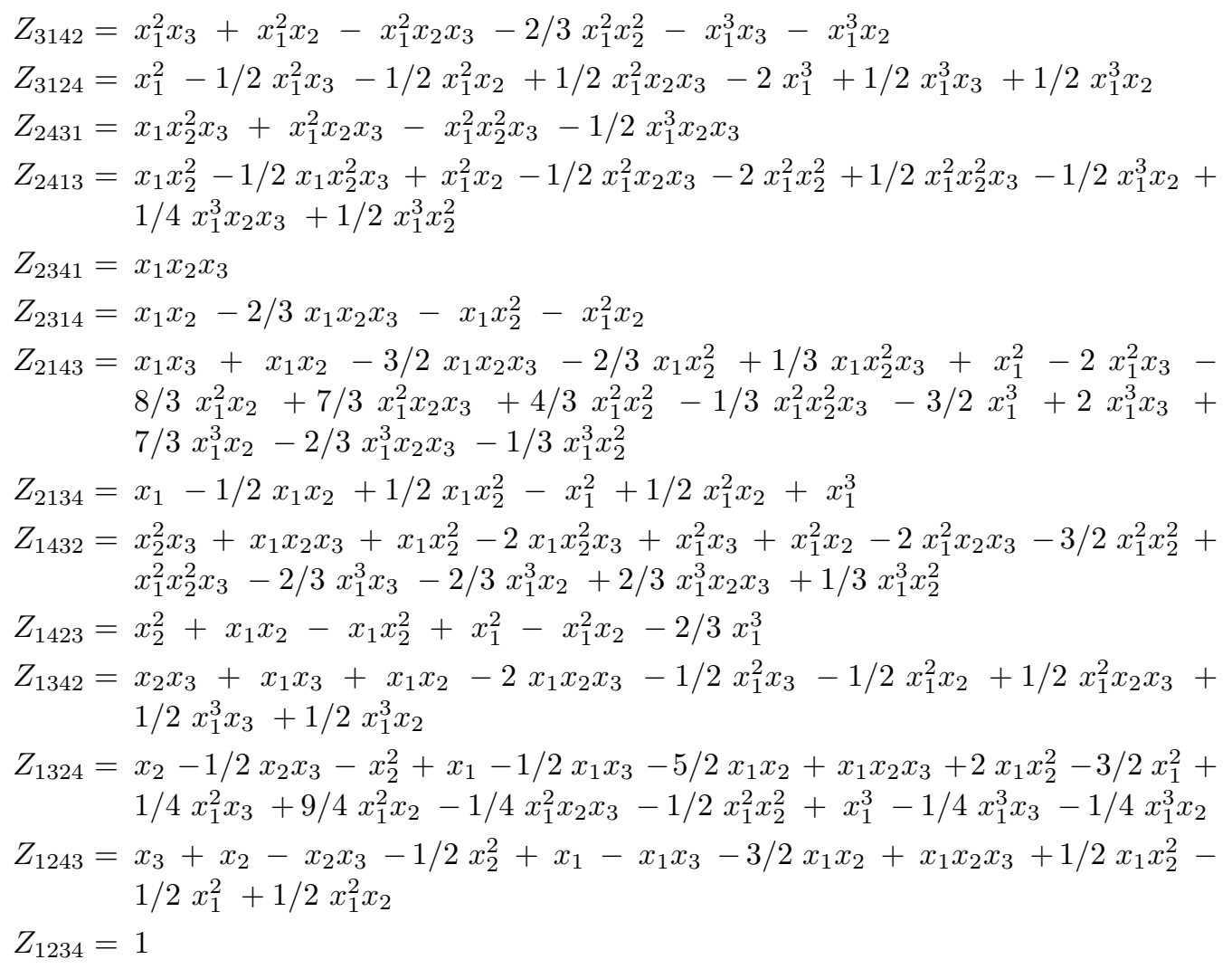

7.2. Adjoint polynomials.

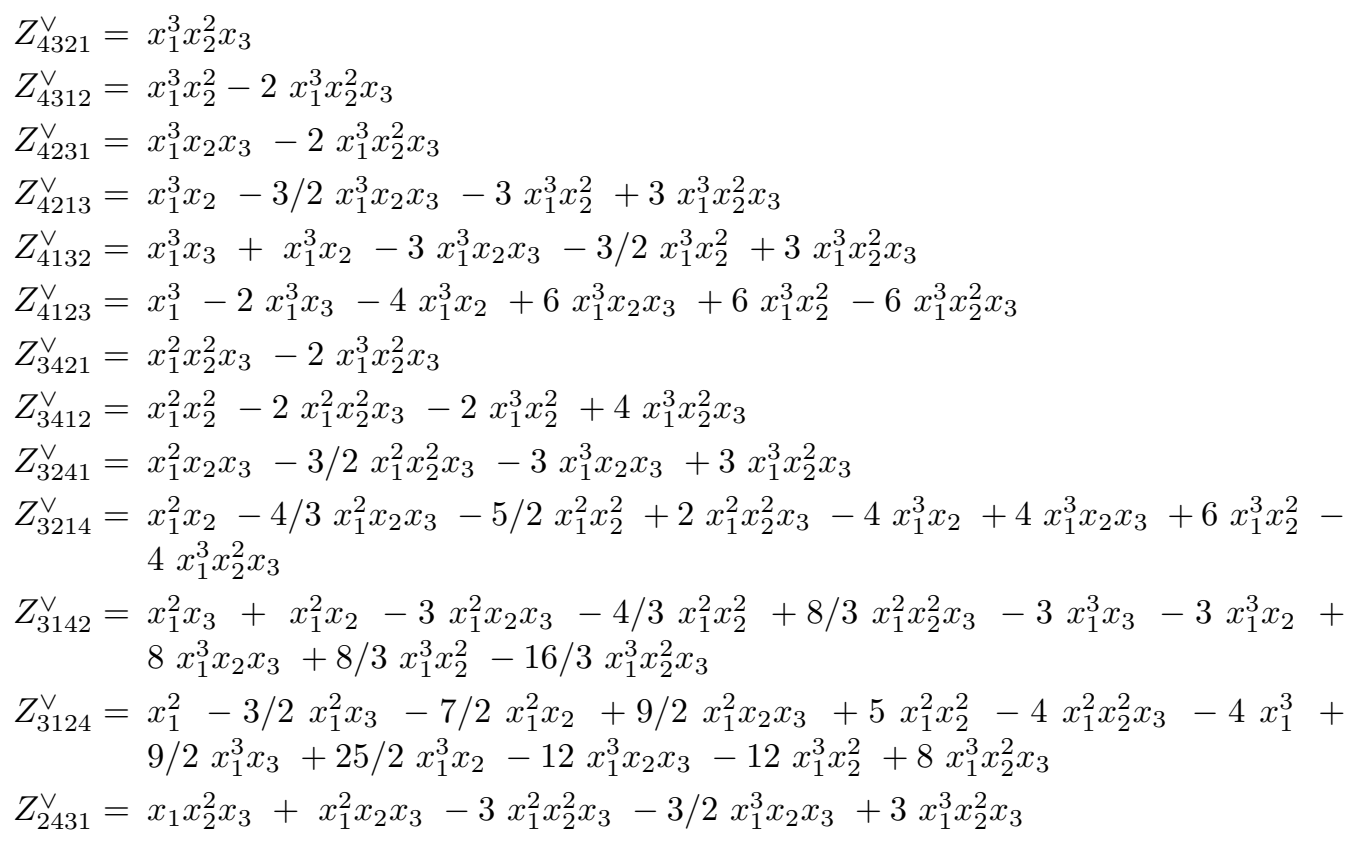




$$
\begin{aligned}
& Z_{2413}^{\vee}=x_{1} x_{2}^{2}-3 / 2 x_{1} x_{2}^{2} x_{3}+x_{1}^{2} x_{2}-3 / 2 x_{1}^{2} x_{2} x_{3}-4 x_{1}^{2} x_{2}^{2}+9 / 2 x_{1}^{2} x_{2}^{2} x_{3}-3 / 2 x_{1}^{3} x_{2}+ \\
& 9 / 4 x_{1}^{3} x_{2} x_{3}+9 / 2 x_{1}^{3} x_{2}^{2}-9 / 2 x_{1}^{3} x_{2}^{2} x_{3} \\
& Z_{2341}^{\vee}=x_{1} x_{2} x_{3}-2 x_{1} x_{2}^{2} x_{3}-4 x_{1}^{2} x_{2} x_{3}+6 x_{1}^{2} x_{2}^{2} x_{3}+6 x_{1}^{3} x_{2} x_{3}-6 x_{1}^{3} x_{2}^{2} x_{3} \\
& Z_{2314}^{\vee}=x_{1} x_{2}-4 / 3 x_{1} x_{2} x_{3}-3 x_{1} x_{2}^{2}+8 / 3 x_{1} x_{2}^{2} x_{3}-5 x_{1}^{2} x_{2}+16 / 3 x_{1}^{2} x_{2} x_{3}+10 x_{1}^{2} x_{2}^{2}- \\
& 8 x_{1}^{2} x_{2}^{2} x_{3}+8 x_{1}^{3} x_{2}-8 x_{1}^{3} x_{2} x_{3}-12 x_{1}^{3} x_{2}^{2}+8 x_{1}^{3} x_{2}^{2} x_{3} \\
& Z_{2143}^{\vee}=x_{1} x_{3}+x_{1} x_{2}-5 / 2 x_{1} x_{2} x_{3}-4 / 3 x_{1} x_{2}^{2}+2 x_{1} x_{2}^{2} x_{3}+x_{1}^{2}-4 x_{1}^{2} x_{3}-16 / 3 x_{1}^{2} x_{2}+ \\
& 9 x_{1}^{2} x_{2} x_{3}+16 / 3 x_{1}^{2} x_{2}^{2}-6 x_{1}^{2} x_{2}^{2} x_{3}-5 / 2 x_{1}^{3}+7 x_{1}^{3} x_{3}+9 x_{1}^{3} x_{2}-12 x_{1}^{3} x_{2} x_{3}- \\
& 6 x_{1}^{3} x_{2}^{2}+6 x_{1}^{3} x_{2}^{2} x_{3} \\
& Z_{2134}^{\vee}=x_{1}-2 x_{1} x_{3}-7 / 2 x_{1} x_{2}+5 x_{1} x_{2} x_{3}+9 / 2 x_{1} x_{2}^{2}-4 x_{1} x_{2}^{2} x_{3}-5 x_{1}^{2}+8 x_{1}^{2} x_{3}+ \\
& 31 / 2 x_{1}^{2} x_{2}-18 x_{1}^{2} x_{2} x_{3}-15 x_{1}^{2} x_{2}^{2}+12 x_{1}^{2} x_{2}^{2} x_{3}+11 x_{1}^{3}-14 x_{1}^{3} x_{3}-26 x_{1}^{3} x_{2}+ \\
& 24 x_{1}^{3} x_{2} x_{3}+18 x_{1}^{3} x_{2}^{2}-12 x_{1}^{3} x_{2}^{2} x_{3} \\
& Z_{1432}^{\vee}=x_{2}^{2} x_{3}+x_{1} x_{2} x_{3}+x_{1} x_{2}^{2}-4 x_{1} x_{2}^{2} x_{3}+x_{1}^{2} x_{3}+x_{1}^{2} x_{2}-4 x_{1}^{2} x_{2} x_{3}-5 / 2 x_{1}^{2} x_{2}^{2}+ \\
& 6 x_{1}^{2} x_{2}^{2} x_{3}-4 / 3 x_{1}^{3} x_{3}-4 / 3 x_{1}^{3} x_{2}+4 x_{1}^{3} x_{2} x_{3}+2 x_{1}^{3} x_{2}^{2}-4 x_{1}^{3} x_{2}^{2} x_{3} \\
& Z_{1423}^{\vee}=x_{2}^{2}-2 x_{2}^{2} x_{3}+x_{1} x_{2}-2 x_{1} x_{2} x_{3}-5 x_{1} x_{2}^{2}+8 x_{1} x_{2}^{2} x_{3}+x_{1}^{2}-2 x_{1}^{2} x_{3}- \\
& 5 x_{1}^{2} x_{2}+8 x_{1}^{2} x_{2} x_{3}+10 x_{1}^{2} x_{2}^{2}-12 x_{1}^{2} x_{2}^{2} x_{3}-4 / 3 x_{1}^{3}+8 / 3 x_{1}^{3} x_{3}+16 / 3 x_{1}^{3} x_{2}- \\
& 8 x_{1}^{3} x_{2} x_{3}-8 x_{1}^{3} x_{2}^{2}+8 x_{1}^{3} x_{2}^{2} x_{3} \\
& Z_{1342}^{\vee}=x_{2} x_{3}-2 x_{2}^{2} x_{3}+x_{1} x_{3}+x_{1} x_{2}-6 x_{1} x_{2} x_{3}-2 x_{1} x_{2}^{2}+8 x_{1} x_{2}^{2} x_{3}-7 / 2 x_{1}^{2} x_{3}- \\
& 7 / 2 x_{1}^{2} x_{2}+25 / 2 x_{1}^{2} x_{2} x_{3}+5 x_{1}^{2} x_{2}^{2}-12 x_{1}^{2} x_{2}^{2} x_{3}+9 / 2 x_{1}^{3} x_{3}+9 / 2 x_{1}^{3} x_{2}- \\
& 12 x_{1}^{3} x_{2} x_{3}-4 x_{1}^{3} x_{2}^{2}+8 x_{1}^{3} x_{2}^{2} x_{3} \\
& Z_{1324}^{\vee}=x_{2}-3 / 2 x_{2} x_{3}-3 x_{2}^{2}+3 x_{2}^{2} x_{3}+x_{1}-3 / 2 x_{1} x_{3}-15 / 2 x_{1} x_{2}+9 x_{1} x_{2} x_{3}+ \\
& 13 x_{1} x_{2}^{2}-12 x_{1} x_{2}^{2} x_{3}-9 / 2 x_{1}^{2}+21 / 4 x_{1}^{2} x_{3}+73 / 4 x_{1}^{2} x_{2}-75 / 4 x_{1}^{2} x_{2} x_{3}-22 x_{1}^{2} x_{2}^{2}+ \\
& 18 x_{1}^{2} x_{2}^{2} x_{3}+6 x_{1}^{3}-27 / 4 x_{1}^{3} x_{3}-75 / 4 x_{1}^{3} x_{2}+18 x_{1}^{3} x_{2} x_{3}+18 x_{1}^{3} x_{2}^{2}-12 x_{1}^{3} x_{2}^{2} x_{3} \\
& Z_{1243}^{\vee}=x_{3}+x_{2}-3 x_{2} x_{3}-3 / 2 x_{2}^{2}+3 x_{2}^{2} x_{3}+x_{1}-5 x_{1} x_{3}-13 / 2 x_{1} x_{2}+14 x_{1} x_{2} x_{3}+ \\
& 15 / 2 x_{1} x_{2}^{2}-12 x_{1} x_{2}^{2} x_{3}-7 / 2 x_{1}^{2}+11 x_{1}^{2} x_{3}+31 / 2 x_{1}^{2} x_{2}-26 x_{1}^{2} x_{2} x_{3}-15 x_{1}^{2} x_{2}^{2}+ \\
& 18 x_{1}^{2} x_{2}^{2} x_{3}+5 x_{1}^{3}-14 x_{1}^{3} x_{3}-18 x_{1}^{3} x_{2}+24 x_{1}^{3} x_{2} x_{3}+12 x_{1}^{3} x_{2}^{2}-12 x_{1}^{3} x_{2}^{2} x_{3} \\
& Z_{1234}^{\vee}=1-2 x_{3}-4 x_{2}+6 x_{2} x_{3}+6 x_{2}^{2}-6 x_{2}^{2} x_{3}-6 x_{1}+10 x_{1} x_{3}+22 x_{1} x_{2}-28 x_{1} x_{2} x_{3}- \\
& 26 x_{1} x_{2}^{2}+24 x_{1} x_{2}^{2} x_{3}+16 x_{1}^{2}-22 x_{1}^{2} x_{3}-48 x_{1}^{2} x_{2}+52 x_{1}^{2} x_{2} x_{3}+44 x_{1}^{2} x_{2}^{2}- \\
& 36 x_{1}^{2} x_{2}^{2} x_{3}-22 x_{1}^{3}+28 x_{1}^{3} x_{3}+52 x_{1}^{3} x_{2}-48 x_{1}^{3} x_{2} x_{3}-36 x_{1}^{3} x_{2}^{2}+24 x_{1}^{3} x_{2}^{2} x_{3}
\end{aligned}
$$

7.3. Transition matrices with Schubert polynomials. The following matrices give the decompositions of the polynomials $Z_{\mu}$ and $Z_{\mu}^{\vee}$ in the basis of Schubert polynomials. Rows and columns are indexed by permutations in reverse lexicographic order:

[4321, 4312, 4231, 4213, 4132, 4123, 3421, 3412, 3241, 3214, 3142, 3124,

$2431,2413,2341,2314,2143,2134,1432,1423,1342,1324,1243,1234$

The bar over a number is to be interpreted as a minus sign. 
7.3.1. $M(Z, X)$. The entry in row $\mu$ and column $\nu$ of the following matrix is equal to the coefficient of $X_{\nu}$ in $Z_{\mu}$. This number is also the coefficient of $Z_{\omega \mu}^{\vee}$ in $X_{\omega \nu}$.

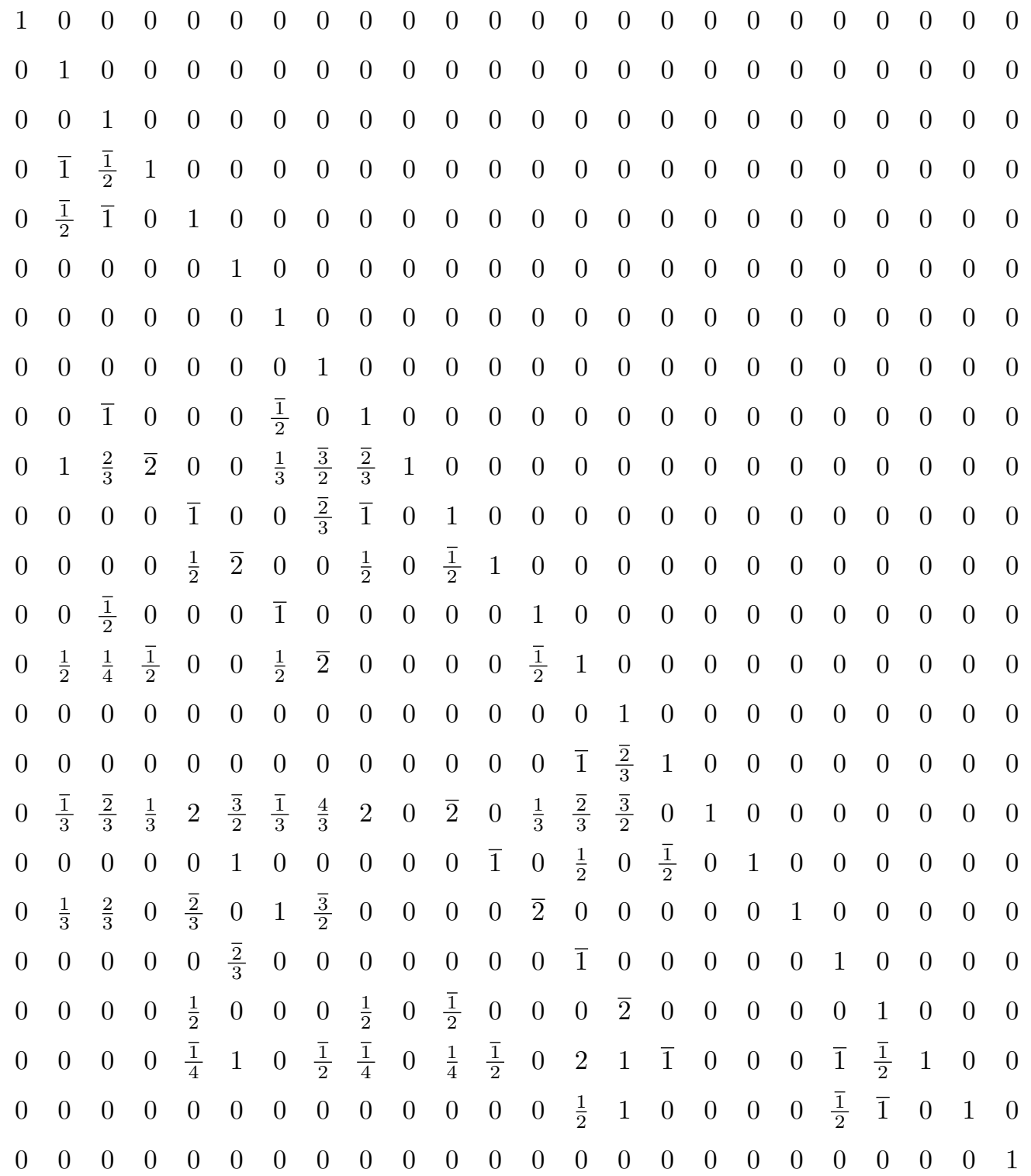


7.3.2. $M\left(Z^{\vee}, X\right)$. The entry in row $\mu$ and column $\nu$ of the following matrix is equal to the coefficient of $X_{\nu}$ in $Z_{\mu}^{\vee}$. This number is also the coefficient of $Z_{\omega \mu}$ in $X_{\omega \nu}$.

$\begin{array}{llllllllllllllllllllllll}1 & 0 & 0 & 0 & 0 & 0 & 0 & 0 & 0 & 0 & 0 & 0 & 0 & 0 & 0 & 0 & 0 & 0 & 0 & 0 & 0 & 0 & 0 & 0\end{array}$

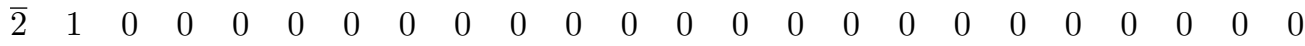

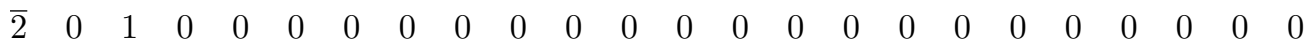

$\begin{array}{lllllllllllllllllllllllll}3 & \overline{3} & \frac{\overline{3}}{2} & 1 & 0 & 0 & 0 & 0 & 0 & 0 & 0 & 0 & 0 & 0 & 0 & 0 & 0 & 0 & 0 & 0 & 0 & 0 & 0 & 0\end{array}$

$\begin{array}{llllllllllllllllllllllll}3 & \frac{\overline{3}}{2} & \overline{3} & 0 & 1 & 0 & 0 & 0 & 0 & 0 & 0 & 0 & 0 & 0 & 0 & 0 & 0 & 0 & 0 & 0 & 0 & 0 & 0 & 0\end{array}$

$\begin{array}{llllllllllllllllllllllll}\overline{6} & 6 & 6 & \overline{2} & \overline{2} & 1 & 0 & 0 & 0 & 0 & 0 & 0 & 0 & 0 & 0 & 0 & 0 & 0 & 0 & 0 & 0 & 0 & 0 & 0\end{array}$

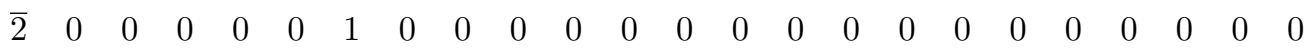

$4 \begin{array}{llllllllllllllllllllllll}4 & 0 & 0 & 0 & 0 & \overline{2} & 1 & 0 & 0 & 0 & 0 & 0 & 0 & 0 & 0 & 0 & 0 & 0 & 0 & 0 & 0 & 0 & 0\end{array}$

$\begin{array}{llllllllllllllllllllllll}3 & 0 & \overline{3} & 0 & 0 & 0 & \frac{\overline{3}}{2} & 0 & 1 & 0 & 0 & 0 & 0 & 0 & 0 & 0 & 0 & 0 & 0 & 0 & 0 & 0 & 0 & 0\end{array}$

$\begin{array}{llllllllllllllllllllllll}\overline{4} & 6 & 4 & \overline{4} & 0 & 0 & 2 & \frac{\overline{5}}{2} & \frac{\overline{4}}{3} & 1 & 0 & 0 & 0 & 0 & 0 & 0 & 0 & 0 & 0 & 0 & 0 & 0 & 0 & 0\end{array}$

$\begin{array}{lllllllllllllllllllllllll}\overline{16} & \frac{8}{3} & 8 & 0 & \overline{3} & 0 & \frac{8}{3} & \frac{\overline{4}}{3} & \overline{3} & 0 & 1 & 0 & 0 & 0 & 0 & 0 & 0 & 0 & 0 & 0 & 0 & 0 & 0 & 0\end{array}$

$8 \begin{array}{llllllllllllllllllllllll}8 & \overline{12} & 8 & \frac{9}{2} & \overline{4} & \overline{4} & 5 & \frac{9}{2} & \overline{2} & \frac{\overline{3}}{2} & 1 & 0 & 0 & 0 & 0 & 0 & 0 & 0 & 0 & 0 & 0 & 0 & 0\end{array}$

$\begin{array}{lllllllllllllllllllllllll}3 & 0 & \frac{\overline{3}}{2} & 0 & 0 & 0 & \overline{3} & 0 & 0 & 0 & 0 & 0 & 1 & 0 & 0 & 0 & 0 & 0 & 0 & 0 & 0 & 0 & 0 & 0\end{array}$

$\begin{array}{llllllllllllllllllllllll}\frac{\overline{9}}{2} & \frac{9}{2} & \frac{9}{4} & \frac{\overline{3}}{2} & 0 & 0 & \frac{9}{2} & \overline{4} & 0 & 0 & 0 & 0 & \frac{\overline{3}}{2} & 1 & 0 & 0 & 0 & 0 & 0 & 0 & 0 & 0 & 0 & 0\end{array}$

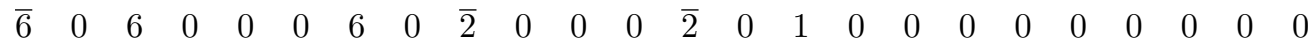

$\begin{array}{llllllllllllllllllllllll}8 & \overline{12} & \overline{8} & 8 & 0 & 0 & \overline{8} & 10 & \frac{8}{3} & \overline{2} & 0 & 0 & \frac{8}{3} & \overline{3} & \frac{\overline{4}}{3} & 1 & 0 & 0 & 0 & 0 & 0 & 0 & 0 & 0\end{array}$

$\begin{array}{lllllllllllllllllllllllll}6 & \overline{6} & \overline{12} & 2 & 7 & \frac{\overline{5}}{2} & \overline{6} & \frac{16}{3} & 7 & 0 & \overline{4} & 0 & 2 & \frac{\overline{4}}{3} & \frac{\overline{5}}{2} & 0 & 1 & 0 & 0 & 0 & 0 & 0 & 0 & 0\end{array}$

$\begin{array}{lllllllllllllllllllllllll}\overline{12} & 18 & 24 & \overline{12} & \overline{14} & 11 & 12 & \overline{15} & \overline{14} & 3 & 8 & \overline{3} & \overline{4} & \frac{9}{2} & 5 & \frac{\overline{3}}{2} & \overline{2} & 1 & 0 & 0 & 0 & 0 & 0 & 0\end{array}$

$\begin{array}{lllllllllllllllllllllllll}\overline{4} & 2 & 4 & 0 & \frac{\overline{4}}{3} & 0 & 6 & \frac{\overline{5}}{2} & 0 & 0 & 0 & 0 & \overline{4} & 0 & 0 & 0 & 0 & 0 & 1 & 0 & 0 & 0 & 0 & 0\end{array}$

$\begin{array}{lllllllllllllllllllllllll}8 & \overline{8} & \overline{8} & \frac{8}{3} & \frac{8}{3} & \frac{\overline{4}}{3} & \overline{12} & 10 & 0 & 0 & 0 & 0 & 8 & \overline{3} & 0 & 0 & 0 & 0 & \overline{2} & 1 & 0 & 0 & 0 & 0\end{array}$

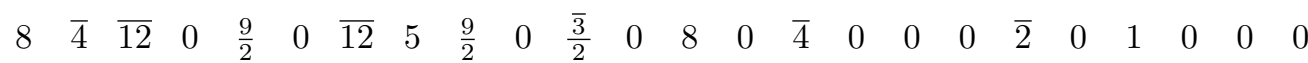

$\begin{array}{llllllllllllllllllllllllllll}\overline{12} & 18 & 18 & \overline{12} & \frac{\overline{27}}{4} & 6 & 18 & \overline{22} & \frac{\overline{27}}{4} & 3 & \frac{9}{4} & \frac{\overline{3}}{2} & \overline{12} & 10 & 6 & \overline{3} & 0 & 0 & 3 & \overline{3} & \frac{\overline{3}}{2} & 1 & 0 & 0\end{array}$

$\begin{array}{lllllllllllllllllllllllll}\overline{12} & 12 & 24 & \overline{4} & \overline{14} & 5 & 18 & \overline{15} & \overline{14} & 0 & 8 & 0 & \overline{12} & \frac{9}{2} & 11 & 0 & \overline{2} & 0 & 3 & \frac{\overline{3}}{2} & \overline{3} & 0 & 1 & 0\end{array}$

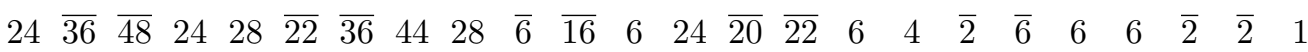




\section{References}

[1] I. N. Bernstein, I. M. Gelfand and S. I. Gelfand, Schubert cells and the cohomology of the spaces $G / P$, Russian Math. Surveys 28 (1973), 1-26.

[2] I. V. Cherednik, On R-matrix quantization of formal loop groups, in: Group theoretical methods in physics, Vol. II (Yurmala, 1985), 161-180, VNU Sci. Press, Utrecht, 1986.

[3] I. V. Cherednik, Quantum groups as hidden symmetries of classic representation theory, in: Differential geometric methods in theoretical physics (A. I. Solomon ed.), World Scientific, Singapore, 1989, 47-54.

[4] M. Demazure, Désingularisation des variétés de Schubert généralisées, Ann. Sci. École Norm. Sup. (4) 7 (1974), 53-88.

[5] M. Demazure, Invariants symétriques entiers des groupes de Weyl et torsion, Invent. Math. 21 (1973), 287-301.

[6] G. Duchamp, D. Krob, A. Lascoux, B. Leclerc, T. Scharf and J.-Y. Thibon, EulerPoincaré characteristic and polynomial representations of Iwahori-Hecke algebras, Publ. Res. Inst. Math. Sci. 31 (1995), 179-201.

[7] W. Fulton, Schubert varieties in flag bundles for the Classical Groups, preprint, University of Chicago, 1994; to appear in: Proceedings of the Conference in Honor of Hirzebruch's 65th Birthday, Bar Ilan, 1993.

[8] F. Hirzebruch, Topological methods in algebraic geometry, Springer, Berlin, 1966.

[9] A. Kerber, A. Kohnert and A. Lascoux, SYMMETRICA, an object oriented computer algebra system for the symmetric group, J. Symbolic Comput. 14 (1992), 195-203.

[10] A. Lascoux, Classes de Chern des variétés de drapeaux, C. R. Acad. Sci. Paris Sér. I Math. 295 (1982), 393-398.

[11] A. Lascoux and M.-P. Schützenberger, Polynômes de Schubert, C. R. Acad. Sci. Paris Sér. I Math. 294 (1982), 447-450.

[12] A. Lascoux and M.-P. Schützenberger, Symmetrization operators on polynomial rings, Functional Anal. Appl. 21 (1987), 77-78.

[13] G. Lusztig, Equivariant K-theory and representations of Hecke Algebras, Proc. Amer. Math. Soc. 94 (1985), 337-342.

[14] I. G. Macdonald, Notes on Schubert polynomials, Publ. LACIM 6, UQAM, Montréal, 1991.

[15] P. Pragacz and J. Ratajski, Formulas for Lagrangian and orthogonal degeneracy loci: the $\tilde{Q}$-polynomials approach, Max-Planck-Institut für Mathematik Preprint 1994; to appear in Compositio Math.

[16] S. Veigneau, SP, a Maple package for Schubert polynomials, Université de Marne-laVallée, 1994.

[17] C. N. Yang, Some exact results for the many-body problem in one dimension with repulsive delta-function interaction, Phys. Rev. Lett. 19 (1967), 1312-1315. 